\title{
Not to Invade: A Better Strategy?
}

Tracheostomy ventilation has been the traditional means of respiratory support for patients who need long-term respiratory support or when mask noninvasive ventilation (NIV) is no longer effective. However, in recent years diurnal mouthpiece NIV has been used to avoid tracheostomy. ${ }^{1,2}$ In this issue or Respiratory CARE, Bach and Martinez ${ }^{3}$ present a retrospective study of Duchenne muscular dystrophy patients with developing chronic respiratory failure who were supported with an NIV regimen that included nocturnal mask NIV, daytime mouthpiece NIV, cough-augmentation therapy, and an oximetry monitoring protocol to support early intervention for respiratory infection, to decrease the risk of hospitalization from respiratory infection.

\section{See the Original Study on Page 744}

Bach and Martinez report excellent long-term results, in survival, avoidance of intubation and tracheostomy, and even changing from tracheostomy to NIV. They report survival as long as 25 years on full-time NIV, ${ }^{3}$ which is certainly a greater lifespan for individuals with Duchenne muscular dystrophy than one would expect without ventilatory support. ${ }^{4}$ However, we do not know whether patients on full-time NIV live longer than those with tracheostomy, as Bach and Martinez had no control group. ${ }^{3}$

It seems unlikely that a randomized controlled trial of fulltime NIV versus tracheostomy ventilation will be performed, because there may not be sufficient equipoise, at least at centers where full-time NIV is available. The sustained benefit of NIV in Duchenne muscular dystrophy partly depends on the maintenance of bulbar function. With prolonged survival, aspiration risk with developing dysphagia ${ }^{5}$ or decreased oromotor function in a portion of this population may also require the clinician to reevaluate the safest and most effective means of respiratory management. ${ }^{6}$

An important question is whether the results from Bach and Martinez $^{3}$ can be generalized. There is some evidence in the study that the answer to that question is no. They report that

Six out-of-state patients placed on NIV by us were intubated for respiratory-infection-associated acute respiratory failure and underwent tracheotomy locally after $12,7,5$, and $<1$ year of full-time NIV, or, in 2 patients, part-time NIV. Three of the 6 died from tube-related causes after 12 years, 11 years, and 2 months of tracheostomy ventilation....
Supporting full-time NIV in the manner that Bach and Martinez did clearly involves a commitment to the support of NIV respiratory management that may not be available at other medical centers. Their success with NIV in Duchenne muscular dystrophy patients, as compared to other centers mentioned in the study, reflects their long experience and NIV program development. Therefore, the challenge for the respiratory care community at large is how to establish on a wider basis the ability to use full-time NIV effectively and avoid failure. Programs to implement these techniques for centers with a range of experience will need to be established to duplicate the success of Bach and Martinez in NIV management of neuromuscular patients. How or whether that will be accomplished remains a question.

Louis J Boitano MSc RRT Joshua O Benditt MD

Division of Pulmonary and Critical Care Medicine Department of Medicine

University of Washington Seattle, Washington

\section{REFERENCES}

1. Bach JR, Alba AS, Saporito LR. Intermittent positive pressure ventilation via mouth as an alternative to tracheostomy for 257 ventilator users. Chest 1993;103(1):174-182.

2. Toussaint M, Steens M, Wasteels G, Soudon P. Diurnal ventilation via mouthpiece: survival in end-stage Duchenne patients. Eur Respir J 2006;28(3):549-555.

3. Bach JR, Martinez D. Duchenne muscular dystrophy: continuous noninvasive ventilatory support prolongs survival. Respir Care 2011;56(6): 744-750.

4. Phillips MF, Quinlivan RC, Edwards RH, Calverley PM. Changes in spirometry over time as a prognostic marker in patients with Duchenne muscular dystrophy. Am J Respir Crit Care Med 2001;164(12):2191-2194.

5. Willig TN, Paulus J, Lacau Saint Guily J, Béon C, Navarro J. Swallowing problems in neuromuscular disorders. Arch Phys Med Rehabil 1994;75(11):1175-1181.

6. Finder JD, Birnkrant D, Carl J, Farber HJ, Gozal D, Iannaccone ST, et al; American Thoracic Society. Respiratory care of the patient with Duchenne muscular dystrophy: ATS consensus statement. Am J Respir Crit Care Med 2004;170(4):456-465.

The authors have disclosed no conflicts of interest.

Correspondence: Louis J Boitano MSc RRT, Division of Pulmonary and Critical Care Medicine, University of Washington Medical Center, 1959 NE Pacific Street, Box 356166, Seattle WA 98195-6166. E-mail: boitano@u.washington.edu.

DOI: 10.4187/respcare.01337 